\title{
OPTIMAL CONTROL OF THERMAL DAMAGE TO TARGETTED REGIONS IN A BIOLOGICAL MATERIAL
}

\author{
F. SCOTT GAYZIK \\ Department of Mechanical Engineering, \\ School of Biomedical Engineering and Sciences, \\ Virginia Tech \\ Blacksburg, VA 24060 \\ fgayzik@vt.edu
}

\author{
ELAINE P. SCOTT \\ Department of Mechanical Engineering, \\ School of Biomedical Engineering and Sciences, \\ Virginia Tech \\ Blacksburg, VA 24060
}

\author{
TAHAR LOULOU \\ Associate Professor \\ Ecole des Mines d'Albi-Carmaux \\ 81013, Albi, FRANCE
}

\begin{abstract}
A numerical technique with potential applications in hyperthermia treatment planning is presented. The treatment is simulated using a 2D transient computational model of the Pennes bioheat equation within an optimization algorithm. The algorithm recovers the heating protocol which will lead to a desired damage field. The relationship between temperature, time and thermal damage is expressed as a first order rate process using the Arrhenius equation. The objective function of the control problem is based on this thermal damage model. The adjoint method in conjunction with the conjugate gradient algorithm is used to minimize the objective function. The results from a numerical simulation show good agreement between the optimal damage field and the damage field recovered by the algorithm. A comparison between the recovered damage field and the commonly used thermal dose is also made.
\end{abstract}

\section{INTRODUCTION}

Hyperthermia, or the exposure of a region of tissue to temperatures above the physiological norm, has been used alone and in conjunction with other modalities such as chemotherapy for many years in the treatment of cancerous tissue. Research has shown that the ability of these treatments to successfully ablate cancerous tumors can be greatly increased via treatment planning. Hyperthermia treatment planning requires not only a model to calculate the temperature history during treatment but also an accepted thermal damage model to predict the magnitude of damage caused during the treatment protocol.

The objective of this research is to develop an optimal control problem which successfully recovers the time dependent surface heat flux needed to create a desired damage field. A flow chart of optimal control problem is shown in Figure 1.

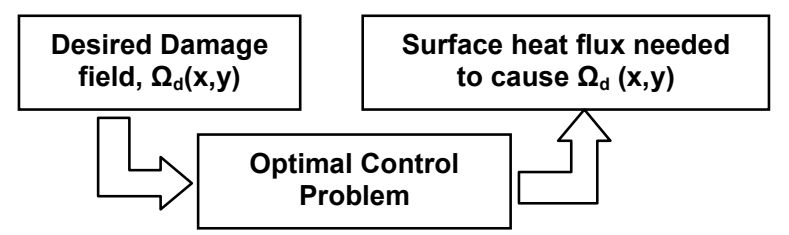

Figure 1: Flow chart of Optimal Control Problem

Figure 2 shows a schematic of the domain over which the computational experiment is run. The control function is the surface heat flux over a portion of the base of a twodimensional domain. The control problem is formulated via the calculus of variations and the conjugate gradient method [1]. 


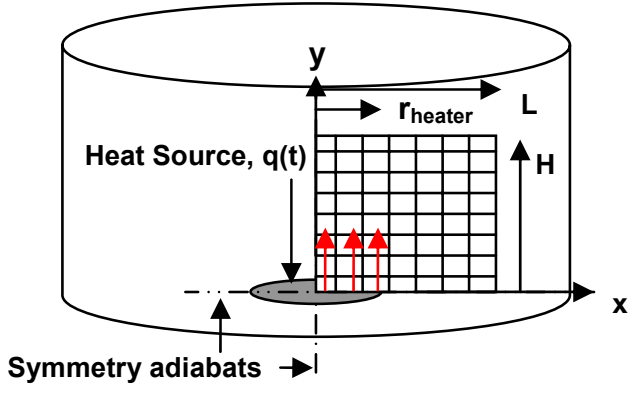

Figure 2: Schematic of mathematical model

The program output of a trial case is presented. The effects of parameterizing of the control function and changing the grid size of the finite difference discretization are also highlighted. While the mathematical model has been developed for regional differences in parameters and temperature dependent thermal properties, a simplified model is considered here so that the results of the optimization program can be verified in a laboratory experiment.

\section{DIRECT PROBLEM FORMULATION}

The control problem presented is an inverse heat transfer problem. In this case the output, the desired damage field, is known but the surface heat flux which would cause this damage field is unknown. To resolve this problem the direct problem is first stated. The direct problem in this case is the Pennes bioheat equation and it is used to calculate the temperature field in the domain based on the current guess of the surface heat flux. The set of differential equations comprising the direct problem are shown below, Eqns. $1-7$. A fully implicit finite difference scheme in radial coordinates was written in MATLAB to calculate the transient temperature field. Please refer to Fig. 2 for a schematic representation of these equations and the finite difference grid. The temperatures at all angular locations of any radius are assumed constant.

$$
\begin{gathered}
C(T) \frac{\partial T(x, y, t)}{\partial t}=k(T) \nabla^{2} T-W\left(T(x, y, t)-T_{\infty}\right) \\
\frac{\partial T(0, y, t)}{\partial x}=\frac{\partial T(L, y, t)}{\partial x}=0 \\
k(T) \frac{\partial T\left(0<x<r_{\text {heater }}, 0, t\right)}{\partial y}=q(t) \\
\frac{\partial T\left(r_{\text {heater }}<x<L, 0, t\right)}{\partial y}=0 \\
\frac{\partial T(x, H, t)}{\partial y}=0, T(x, y, 0)=T_{\infty}
\end{gathered}
$$

\section{OBJECTIVE FUNCTION}

The thermal damage is modeled as a first-order rate process using the Arrhenius equation. [2]

$$
\Omega(x, y)=\ln \frac{C(0)}{C\left(t_{f}\right)}=A \int_{0}^{t_{f}} \exp \frac{-E_{a}}{R T(x, y, \tau)} d \tau
$$

Here $\mathrm{A}$ is the molecular frequency factor, $\mathrm{E}_{\mathrm{a}}$ is the activation energy and $\mathrm{R}$ is the universal gas constant. $\mathrm{C}$ is the concentration of undamaged material at a specified time. If $\mathrm{C}(0)$ is taken to be $100 \%$, then $\Omega(\mathrm{x}, \mathrm{y})=1$ correlates to $\mathrm{C}\left(\mathrm{t}_{\mathrm{f}}\right)=$ $63.2 \%$, which is the concentration of damaged material in a control volume at the end of a heating protocol. An optimized damage field would result in targeted ablation areas with $\Omega>1$. The objective functional to be minimized is the difference between the damage field resulting from the current guess of the heat flux and the optimal damage field. It is stated below.

$$
J(q(t))=\iint_{x, y}\left[\int_{0}^{t} \exp \frac{-E_{a}}{R T(x, y, \tau ; q(t))} d \tau-\Omega_{d}(x, y)\right]^{2} d x d y
$$

The subscript $d$ denotes the desired damage field and the $q(t)$ states that the temperature field in this calculation is based on the current value of the control function.

\section{VARIATION PROBLEM FORMULATION}

The control problem formulation requires that we know how the system will react to small changes in the independent variable. If we let $\Delta \mathrm{T}(\mathrm{x}, \mathrm{y}, \mathrm{t})$ be a small change in the temperature field due to a perturbation of the control function, $\mathrm{q}(\mathrm{t})$ this variation can be shown mathematically as:

$$
\begin{aligned}
& \Delta T(x, y, t) \approx T(x, y, t: q(t)+\Delta q)-T(x, y, t ; q(t)) \\
& \Delta T(x, y, t) \approx T_{\mathcal{E}}(x, y, t ; q(t))-T(x, y, t ; q(t))
\end{aligned}
$$

The perturbed temperature field is found by solving the variation problem, the mathematical definition of which is shown below in Eqns. (11-17). This set of differential equations is developed by substituting the variation of the temperature shown in Eqn. 10 to the direct problem and applying a limiting process $\epsilon \longrightarrow 0$.

$$
\begin{gathered}
C(T) \frac{\partial \Delta T(x, y, t)}{\partial t}=k(T) \nabla^{2}(\Delta T)-W(\Delta T(x, y, t)) \\
\frac{\partial \Delta T(0, y, t)}{\partial x}=\frac{\partial \Delta T(L, y, t)}{\partial x}=0 \\
k(T) \frac{\partial \Delta T\left(0<x<r_{\text {heater }}, 0, t\right)}{\partial y}=\Delta q(t) \\
\frac{\partial \Delta T\left(r_{\text {heater }}<x<L, 0, t\right)}{\partial y}=0 \\
\frac{\partial \Delta T(x, H, t)}{\partial y}=0, \quad \Delta T(x, y, 0)=T_{\infty}
\end{gathered}
$$

By using the same limiting process, we can find the variation of the objective function.

$$
\begin{aligned}
& \Delta J(q) \\
& =\frac{2 A E_{a}}{R} \int_{o}^{L} \int_{0}^{H_{f}} \int_{0}^{t_{f}}\left[\Omega_{c}(x, y ; q)-\Omega_{d}(x, y)\right] \operatorname{Exp} \frac{-E_{a}}{R T(x, y, t)} \frac{\Delta T(x, y, t)}{T(x, y, t)^{2}} d t d y d x
\end{aligned}
$$

\section{ADJOINT PROBLEM FORMULATION}

The functional gradient is found via the adjoint problem. This problem is a set of differential equations shown below, Eqns. (19-25). The adjoint problem is found by analyzing the stationary conditions of the variation of the Lagrangian of the system. For further development see [1]. 


$$
\begin{gathered}
-C(T) \frac{\partial \psi(x, y, t)}{\partial t}=k(T) \nabla^{2}(\psi)-W(\psi(x, y, t))+Z(x, y, t) \\
\frac{\partial \psi(0, y, t)}{\partial x}=\frac{\partial \psi(L, y, t)}{\partial x}=0 \\
\frac{\partial \psi(x, 0, t)}{\partial y}=\frac{\partial \psi(x, H, t)}{\partial y}=0 \\
\psi\left(x, y, t_{f}\right)=0
\end{gathered}
$$

Where the source term in Eqn. 19 is given below.

$$
\begin{aligned}
& Z(x, y, t) \\
& =\frac{2 A E_{a}}{R}\left[\Omega_{c}(x, y ; q)-\Omega_{d}(x, y)\right] \operatorname{Exp} \frac{-E_{a}}{R T(x, y, t)} \frac{1}{T(x, y, t)^{2}}
\end{aligned}
$$

The solution of this set of equations, $\psi(\mathrm{x}, \mathrm{y}, \mathrm{t})$ can be used to find the gradient of the functional as shown in Eqn. 26.

$$
J^{\prime}(t)=\int_{0}^{r_{\text {heater }}} \psi(x, 0, t) d x
$$

\section{MINIMIZATION PROCEDURE}

With a method to find the gradient of the functional established, the conjugate gradient method can be used to find the heat flux which minimizes the objective function. This method works by choosing successive values of the control function such that the value of Eqn. 9 is continually reduced to a minimum. The adjoint problem and variation problem are solved each iteration, however since both equations are of the same form as the objective function the same finite difference solver can be used for all three problems, making the method more computationally efficient. Convergence is reached when successive estimates of the surface heat flux vector produced no change. See [1] for further details on the minimization procedure.

\section{RESULTS}

The material chosen for the model was albumen, also known as egg white; the thermal and Arrhenius properties used in the model are found Table 1. Albumen was chosen because it has been used previously in research as a tissue phantom and can be used in a laboratory to validate the simulation results $[3,5]$.

The MATLAB code was run over a small spatial domain to reduce computational time, $0.5 \mathrm{~cm} \times 0.5 \mathrm{~cm}$. Twenty control volumes were used in each direction and the simulated treatment protocol lasted 30 minutes. Figure 3 shows the heat flux recovered by the program.

Table 1. Thermal and Arrhenius Properties of Albumen

\begin{tabular}{|c|c|c|c|}
\hline $\mathrm{k}$ & $0.56 \mathrm{~W} / \mathrm{m} / \mathrm{K}$ & $\rho$ & $997 \mathrm{~kg} / \mathrm{m}^{3}$ \\
\hline $\mathrm{Cp}$ & $4180 \mathrm{~J} / \mathrm{kg} / \mathrm{K}$ & $\mathrm{W}$ & $4180 \mathrm{~W} / \mathrm{m}^{3} / \mathrm{K}$ \\
\hline $\mathrm{Ea}$ & $3.85 \mathrm{E}+5 \mathrm{~J} / \mathrm{mol}$ & $\mathrm{A}$ & $3.8 \mathrm{E}+571 / \mathrm{s}$ \\
\hline
\end{tabular}

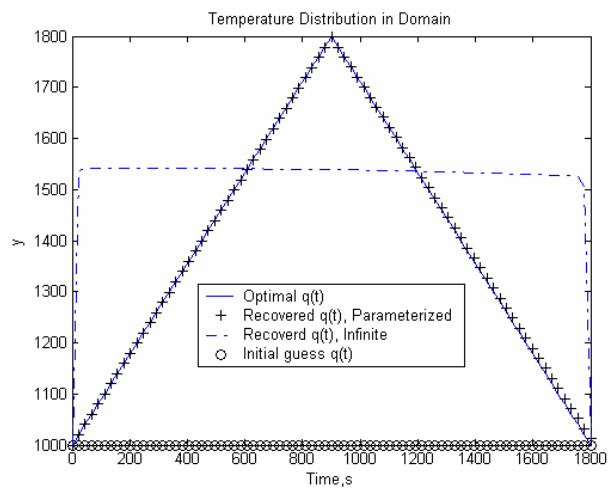

Figure 3: Recovered Heat flux

The recovered damage field and the difference between the recovered and optimal damage fields are shown in Figures 4 and 5.

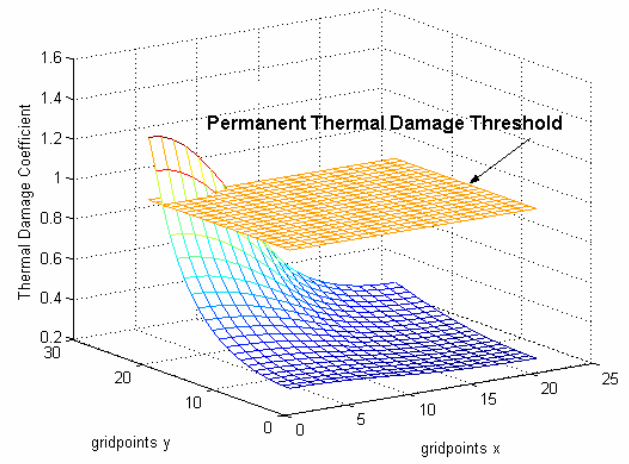

Figure 4: Recovered optimal damage field

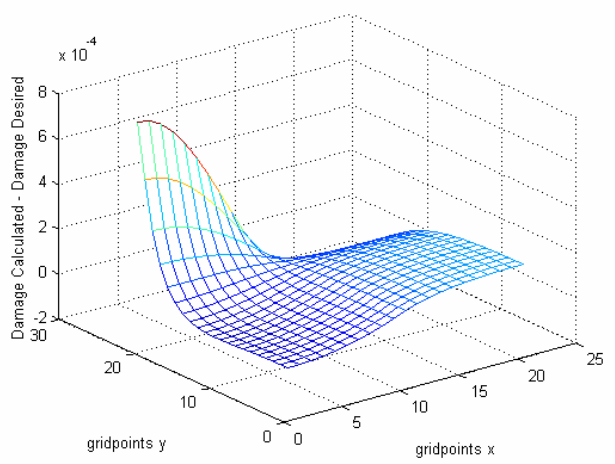

Figure 5: Difference between recovered thermal damage field and optimal thermal damage field

\section{DISCUSSION}

The control problem can be solved in both finite and infinite dimensional form. Figure 3 shows the recovered heat flux and how it differs from the optimal heat flux depending on how the control problem is cast. The optimal heat flux was used to create the desired damage field. Since the solution to problem is not unique, meaning that more than one heat flux function exists which will cause identical damage fields, these forms will give different results. Clearly the finite dimensional form provides a more accurate estimation of the optimal heat 
flux. In this case, $\mathrm{q}(\mathrm{t})$ is parameterized using cubic spline basis functions, $\mathrm{B}^{4}(\mathrm{t})$ as shown below.

$$
q(t)=\sum_{i=1}^{M} a_{i} B_{i}^{4}(t)
$$

The results shown are for $M=3$. However convergence is slower for the finite dimensional form. Figure 4 shows thermal damage field as well as the threshold for permanent thermal damage. Only a small portion of the domain near the heater where $\Omega>1$ was permanently damaged in this simulation. Figure 5 shows the difference between the true optimal damage field and the recovered field. The biggest discrepancy comes near the heater, which should be expected since the temperature gradients will be steepest here. But the accuracy of the method is shown as the difference is on the order of $0.01 \%$ of the recovered damage coefficient field.

It should be noted that the discretization has a large effect on the accuracy of damage coefficient and the finite difference calculations. Convergence will not be reached if too coarse a discretization is used, particularly near the source. In this simulation, a uniform grid with $\Delta \mathrm{x}$ and $\Delta \mathrm{y}=0.25 \mathrm{~mm}$ was used. The time step used was $\Delta t=22.5 \mathrm{~s}$. The initial guess of the unknown heat flux strongly influences convergence as well.

Loulou and Scott published work on a similar control problem for hyperthermia treatment planning, however the objective function was based on a thermal dose instead of a thermal damage coefficient $[1,4]$. The thermal dose was developed as a way of quantifying a hyperthermia treatment by converting it to an amount of equivalent minutes at an arbitrary reference temperature, $T_{r}=43^{\circ} \mathrm{C}$. The equation for the thermal dose is given below.

$$
D_{43}=\int_{t=0}^{t_{f}} R_{d}^{43-T(x, y, t)} d t
$$

Research has shown that $R_{d}$ ranges from 0.4 to 0.8 above $43^{\circ} \mathrm{C}$ and is roughly half that value below $43^{\circ} \mathrm{C}$. While no values of $R_{d}$ specifically for albumen were found in the literature, the most common values for $\mathrm{R}$ in biological systems were used. These are $R_{d}=0.5$ above $43^{\circ} \mathrm{C}$ and 0.25 below $43^{\circ} \mathrm{C}$ [4]. $R_{d}$ is also allowed to vary linearly around the reference temperature as shown in Figure 6, where $\epsilon=0.5$ [1].

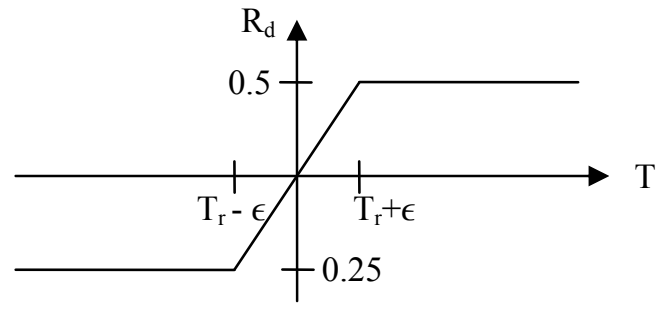

Figure 6. Linearization of $R_{d}$ value about $T_{r}$

The transient temperature history resulting from the optimal heating protocol, shown in Fig. 3, was used in calculating the corresponding thermal dose. The resulting thermal dose field is shown in Fig. 7. The results of these simulations allow for comparison of the thermal dose and thermal damage models. The damage model has a threshold over which permanent thermal damage occurs, $\Omega=1$. By comparing Fig. 7 and Fig. 4 one can show the amount of equivalent minutes at $43^{\circ} \mathrm{C}$ which would cause permanent damage for the given material. Future work will optimize the surface heat flux with respect to the thermal dose and thermal damage coefficient for the same constraints and see how, if at all, the optimal heat flux differs for these cases.

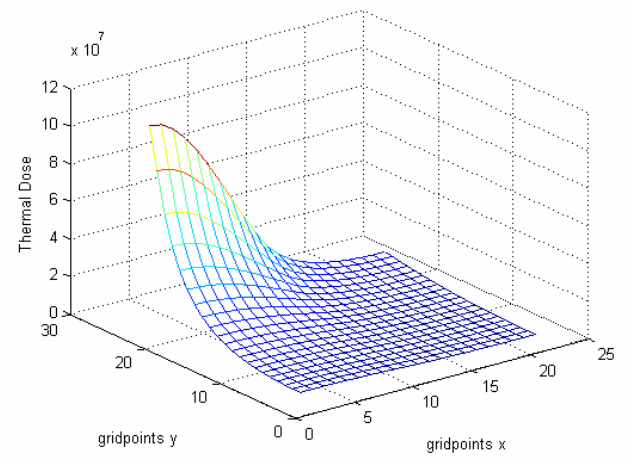

Figure 7. Thermal dose due to optimal heating protocol

\section{CONCLUSIONS}

An optimal control algorithm was developed which recovers the heat flux required to cause a desired thermal damage field within a specified domain. The algorithm has potential applications in hyperthermia where a need for accurate and flexible treatment planning exists. The control problem outlined here can be modified to include regional differences in thermal properties but is also simple enough to validate in a laboratory setting.

\section{REFERENCES}

1. Loulou T. and Scott, E.P., 2002, Thermal Dose Optimization in Hyperthermia Treatments by Using the Conjugate Gradient Method, Numerical Heat Transfer, Part A, Vol 42, $\mathrm{N}^{\circ} 7$, pp. 661-683.

2. Pearce. J.A., Thomen, S., 1992, Kinetic models of tissue fusion processes, Proc. SPIE, Vol 1643, pp. 251-260.

3. Pfefer T.J., et al., 2000, Dynamics of Pulsed Holmium: YAG Laser Photocoagulation of Albumen, Phys. Med. Biol, Vol 45, pp. 1099-1114.

4. Sapareto, S.A., Dewey, W.D., 1984, Thermal Dose Determination in Cancer Therapy, Int. J. Radiation Oncology, Biol., Phys., Vol 10, pp. 787-800.

5. Yang. Y, Welsh, A. J., 1991, Rate Process Parameters of Albumen, Lasers Surg. Med., Vol 11, pp. 188-190 